Check for updates

Cite this: RSC Adv., 2017, 7, 36516

\title{
Dense AuNP/MoS 2 hybrid fabrication on fiber membranes for molecule separation and SERS detection
}

\author{
Yuefeng Zhao, $\dagger^{a}$ Xiaoxiao Pan, $\mathbb{D} \dagger^{\mathrm{a}}$ Liren Zhang, ${ }^{\mathrm{b}}$ Yuanyuan $\mathrm{Xu},{ }^{a}$ Chonghui $\mathrm{Li},{ }^{a}$ \\ Jundong Wang, ${ }^{a}$ Jiayu Ou, ${ }^{a}$ Xianwu Xiu, ${ }^{\text {ac }}$ Baoyuan Man*a and Cheng Yang (DD *ac
}

Synthesis of uniform and dense metal nanostructures on the whole exposed fiber, instead of simply on the membrane surface, is necessary for controllable separation and sensitive SERS detection. Herein, uniform and dense AuNP/MoS 2 hybrids were synthesized on single 3D glass fiber (GF) surfaces, obtained by the reaction of a MoS 2 nanosheet and $\mathrm{HAuCl}_{4}$. The chemical mechanism (CM) of the $\mathrm{MoS}_{2}$ layers and the electromagnetic enhancement (EM) of the dense AuNPs were used to enhance the SERS sensitivity. The molecule-separation ability of the obtained GF membranes, constructed by numerous compacted fibers with dense AuNP/MoS 2 hybrids, was not destroyed, and herein, these membranes were used to separate mixed molecules. These $\mathrm{MoS}_{2}$-based hybrid substrates show great potential for excellent paper-based separation and are sensitive SERS substrates for biological and chemical detection.

Received 17th May 2017

Accepted 6th July 2017

DOI: $10.1039 / \mathrm{c} 7 \mathrm{ra05568d}$

rsc.li/rsc-advances

to its features such as simple sample collection, less timeconsumption, and pre-analytical treatment. Paper chromatography is a technique that is used for separating target analytes from complex samples, allowing the definite and unambiguous identification of individual analytes from the sample. The combination of paper separation and SERS detection becomes an effective technique. For example, Hyukjin Jung reported the use of cellulose fiber decorated with silver nanoislands for the separation and detection of small molecules. ${ }^{13}$ Further enhancements in the SERS sensitivity and separation ability are useful for the application of this technique.

Recent studies indicate that $\mathrm{MoS}_{2}$ can be an ideal platform to support SERS activity because of its many advantages. First, $\mathrm{MoS}_{2}$ can provide chemical enhancement and efficient adsorption for various target molecules; hence, the sensitivity of the substrate is enhanced. Second, $\mathrm{MoS}_{2}$ has chemical stability and can be obtained at low temperatures; these features endow the substrate with a long lifetime and effectively reduce energy consumption. ${ }^{14,15}$ Third, the $\mathrm{MoS}_{2}$ nanosheet exhibits high surface area that makes it a promising supporting material to stabilize metal nanoparticles and form hierarchical composites. ${ }^{16}$

Note that AuNPs can be grown in situ on the surface of $\mathrm{MoS}_{2}$ nanosheets by a spontaneous redox reaction with tetrachloroauric acid $\left(\mathrm{HAuCl}_{4}\right)$ without any reducing agent. ${ }^{17,18}$ The density and diameter of the AuNPs can be controlled by changing the conditions of the reaction between the $\mathrm{MoS}_{2}$ nanosheet and $\mathrm{HAuCl}_{4}$ to acquire the best SERS-active substrate. AuNPs can concentrate light into small volumes; this greatly enhances the local electromagnetic field near the metal nanostructures. ${ }^{19}$ The areas with greatly enhanced EM field become hot spots for surface-enhanced Raman

\footnotetext{
${ }^{a}$ School of Physics and Electronics, Shandong Normal University, Jinan 250014, People's Republic of China. E-mail: chengyang@sdnu.edu.cn; byman@sdnu.edu.cn ${ }^{b}$ College of Information Technology, UAE University, Al-Ain, United Arab Emirates 'Institute of Materials and Clean Energy, Shandong Normal University, Jinan 250014, People's Republic of China

$\dagger$ These authors contributed equally.
} 
spectroscopy. ${ }^{20}$ The localized surface plasmon resonance (LSPR) generated on AuNPs induces strong plasmon-exciton coupling, resulting in strong light-matter interaction, thereby enabling enhanced Raman scattering. ${ }^{21}$ In addition, AuNPs are well known for their excellence in biocompatibility and less susceptible to oxidation than most of the other SERS-active nanoparticles. ${ }^{22,23}$

Glass fiber (GF) membrane is a good candidate for paperbased SERS substrate as its inner 3D structure is constructed by numerous compacted fibers, with a fine capillary structure, and it is tolerant to biochemical or chemical circumstances. ${ }^{24}$ Compared to monolithic substrates, the glass fiber offers a large surface area for adsorption due to its 3D structure and immobilizes SERS-active nanoparticles onto the interior surface of the capillary to avoid floating of the nanoparticle aggregates on the surface; this can highly improve reproducibility, enhance Raman signals of molecules, and extend its application. ${ }^{25,26}$ In addition, GF membranes composed of intricate fibers allow paper chromatographic separation under the action of capillary effect, which are feasible for the separation and preconcentration of analytes from a complex substrate. Paper, as a substrate, has been used for the separation and detection of complex samples. However, the structure of paper is not sufficiently uniform; this reduces the speed of sample separation. Moreover, paper as a substrate cannot withstand high temperatures; this limits its scope of application. In this study, GF with a relatively uniform structure and high temperature resistance provided unique capabilities for the two-dimensional chromatographic separation and SERS detection.

In this study, we selected crystal violet (CV) and toluidine blue (TB) as probe molecules to analyze the performance of the substrate. As is known, CV and TB dyes are much studied fluorescent probe molecules with numerous applications in biology and biochemistry. CV and TB have different polarities; hence, they can be spatially isolated on the GF substrate based on their different affinities for the stationary and mobile phases. ${ }^{27}$ We introduced a highly practical and sensitive sensor, GF$\mathrm{MoS}_{2} @ A u N P$ substrate, that provided a new platform for the label-free screening and ultrasensitive detection of the mixture of CV and TB molecules.

\section{Methods}

\section{Reagents and materials}

All chemicals, including ammonium tetrathiomolybdate $\left(\mathrm{NH}_{4}\right)_{2} \mathrm{MoS}_{4}$, dimethylformamide (DMF), chloroauric acid $\left(\mathrm{HAuCl}_{4}\right)$, crystal violet (CV), toluidine blue (TB), ethanol $(99.7 \%)$, and glass fiber, were directly used without further purification.

\section{Preparation of the GF-MoS ${ }_{2} @ A u N P$ substrate}

Large-scale growth of few-layered $\mathrm{MoS}_{2}$ was achieved by thermally decomposing the precursor ammonium tetrathiomolybdate $\left(\left(\mathrm{NH}_{4}\right)_{2} \mathrm{MoS}_{4}\right)$. High-purity $\left(\mathrm{NH}_{4}\right)_{2} \mathrm{MoS}_{4}(0.01 \mathrm{~g})$ was added to $1 \mathrm{~mL}$ dimethylformamide (DMF) to form a solution. The solution was sonicated for $40 \mathrm{~min}$ before use. A glass fiber was immersed in the $\left(\mathrm{NH}_{4}\right)_{2} \mathrm{MoS}_{4}$ solution and then naturally dried.

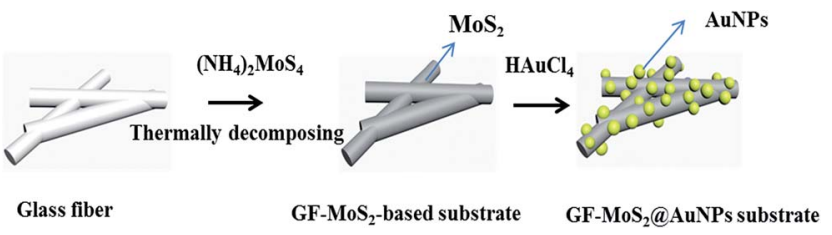

Fig. 1 Schematic of the synthesis of the GF-MoS 2 aAuNP substrate. The precursor $\left(\mathrm{NH}_{4}\right)_{2} \mathrm{MoS}_{4}$ was dip-coated on the substrate. The thermal decomposing process was performed in a quartz tube furnace. AuNPs grew in situ on the surface of the $\mathrm{MoS}_{2}$ nanosheet by a spontaneous redox reaction between the $\mathrm{MoS}_{2}$ nanosheet and tetrachloroauric acid $\left(\mathrm{HAuCl}_{4}\right)$.

The prepared glass fiber was placed in a tube furnace under a flow of a gas mixture of $\mathrm{Ar} / \mathrm{H}_{2}$ at the flow rate $=4 / 1 \mathrm{sccm} . \mathrm{MoS}_{2}$ was formed on the glass fiber via thermal annealing at $500{ }^{\circ} \mathrm{C}$ for 90 min. Raman spectra were obtained via the HORIBA HR800 Raman microscopy system (HORIBA, Kyoto, Japan) (laser wavelength $532 \mathrm{~nm}$, objective lens magnification 50, and laser power $1 \%$ ) to determine the presence of $\mathrm{MoS}_{2}$. AuNP-decorated $\mathrm{MoS}_{2}$ nanocomposite was synthesized via a direct reaction between $\mathrm{MoS}_{2}$ and $\mathrm{HAuCl}_{4}$. The morphology of the sample was characterized using a scanning electron microscope (SEM, Zeiss Gemini Ultra-55, Carl Zeiss, Inc., Oberkochen, Germany). The complete process for the construction of the GF-MoS ${ }_{2} @ A u N P$ substrate is shown in Fig. 1.

\section{Separation of the mixture}

Crystal violet (CV) and toluidine blue (TB) solutions were prepared using deionized water and spotted separately on the glass fiber using a capillary tube with a volume of $5 \mu \mathrm{L}$. The mixed solution of CV and TB was then spotted on the glass fiber using the same procedure. Paper separation was developed using $99.7 \%$ ethanol as a mobile phase. After being dried under ambient conditions, the glass fiber was suspended in a beaker with ethanol for pre-saturation. Pre-saturation took about $15 \mathrm{~min}$. Then, the bottom of the glass fiber was immersed in ethanol. The separation effect of the mixed molecules was analyzed via colorimetric detection of dye molecules.

\section{Results and discussion}

GF membranes were used as substrates for the growth of $\mathrm{MoS}_{2}$ by the thermal decomposition method due to their high temperature resistance. Chemical reduction between $\mathrm{MoS}_{2}$ and $\mathrm{HAuCl}_{4}$ realizes the deposition of dense AuNPs on the glass fiber. The GF-MoS $\mathrm{M}_{2} @ A u N P$ substrate allows highly sensitive detection of $\mathrm{CV}$ and $\mathrm{TB}$ molecules, and its morphology is characterized by SEM. The SEM images of the glass fiber, glass fiber with $\mathrm{MoS}_{2}$, and gold nanoparticle-loaded glass fiber are shown in Fig. 2. Fig. 2a displays the morphology of the glass fiber at different magnifications, and the inset shows the magnified SEM image. The GF membrane is formed by many wire-type glass fibers with different diameters that can trap the precursor and act as the initial nucleation centers for the growth of the AuNPs. In addition, a relatively uniform color is obtained, 

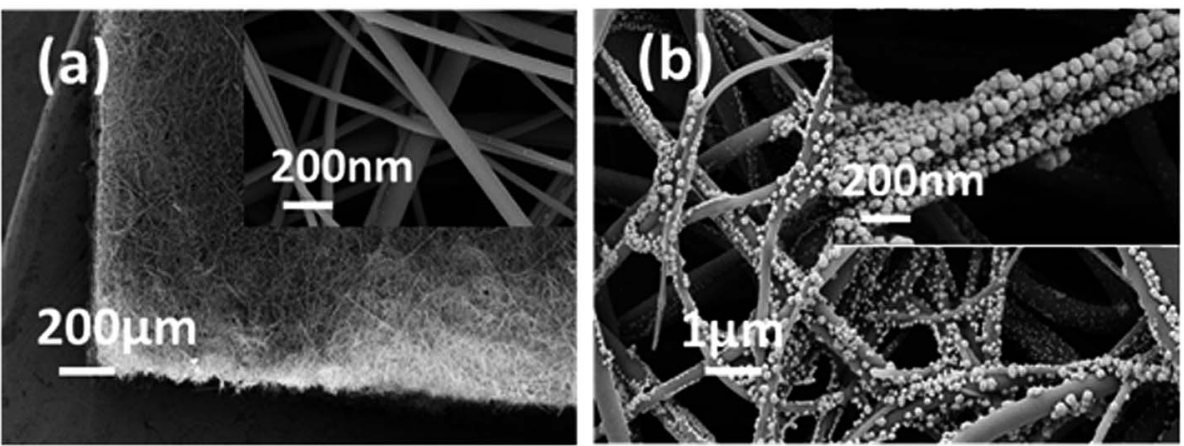

Fig. 2 (a) SEM images of the GF membranes at different magnifications. (b) SEM images of the GF-MoS ${ }_{2}$ (AANP substrate. The inset is the SEM image of the GF-MOS 2 (AuNP substrate at high magnification.

and no rippled or wrinkled structures are detected on each glass fiber. Fig. $2 \mathrm{~b}$ is the SEM image of the GF-MoS $\mathrm{S}_{2}$ @AuNP substrate, and a large number of AuNPs (about $50 \mathrm{~nm}$ in diameter) attached to the GF membranes can be clearly seen in the inset.

Fig. 3 illustrates the numerical results of Raman spectra with peaks at $377 \mathrm{~cm}^{-1}$ and $408 \mathrm{~cm}^{-1}$ attributed to $\mathrm{E}_{2 \mathrm{~g}}^{1}$ and $\mathrm{A}_{1 \mathrm{~g}}$, respectively. The $E_{2 g}^{1}$ (in-plane) mode results from opposite vibrations of two $S$ atoms with respect to the Mo atom, and the $A_{1 g}$ mode is associated with the out-of-plane vibration of $\mathrm{S}$ atoms in opposite directions. ${ }^{28}$ After successful synthesis of the GF$\mathrm{MoS}_{2} @ A u N P$ substrate, we obtained the Raman spectrum of $\mathrm{MoS}_{2}$ on the GF membranes (red line) and GF-MoS ${ }_{2}$ @AuNPs (black line). In Fig. 3a, it can be observed that the Raman signals of $\mathrm{MoS}_{2}$ molecules slightly increase when it is decorated with AuNPs; this indicated a better enhancement effect of the AuNPs. In the following Raman analysis, CV and TB solutions with different concentrations were used to evaluate the SERS performance of the substrate. In addition to the concentration, the absolute amount of the analyte greatly influences the Raman spectra of the substrate. Therefore, we used analytes with the same volume in each Raman analysis. To operationalize rapid diagnosis tests in clinics, all Raman spectra were obtained by adding traces of analytic samples instead of conventional immersion in solution for long periods of time. No characteristic scattering peaks of CV can be observed except the strong fluorescent background on GF membranes, as shown in Fig. 3b. Both the GF-MoS $@$ @AuNP and $\mathrm{MoS}_{2}$ substrates show the Raman enhanced effect toward the CV molecule. All these three substrates show Raman intensity at 914 $\mathrm{cm}^{-1}$ and $1620 \mathrm{~cm}^{-1}$ toward $\mathrm{CV}$; this results indicates that GF$\mathrm{MoS}_{2} @ A u N P$ substrate displays the best SERS performance. The synergies of charge-transfer, plasmon and exciton, and dipoledipole coupling of the $\mathrm{MoS}_{2}$ film cause enhancement in the SERS signal. Therefore, the substrate with $\mathrm{MoS}_{2}$ shows better SERS effect as compared to the blank GF membranes. However, it is worth noting that the SERS intensity of the GF-MoS ${ }_{2}$ @AuNP substrate is much stronger than that of the $\mathrm{MoS}_{2}$ substrate. The presence of AuNPs greatly enhances the local electromagnetic (EM) field near the nanoparticles. In addition, AuNPs can excite surface plasmons and produce strong scattering spectra, thus amplifying the Raman signal. Therefore, the CV molecule on the GF-MoS ${ }_{2} @ A u N P$ substrate displays the most intense characteristic scattering peaks.

The density and size of the AuNPs can be easily controlled by changing the reaction time and keeping other conditions unaltered, which influence the SERS properties of the substrate. We chose $3 \mathrm{~min}$ as a time interval and increased the time of the reaction between $\mathrm{MoS}_{2}$ and $\mathrm{HAuCl}_{4}$ from 3 to 15 min. Fig. 4 shows the SEM images of the GF-MoS ${ }_{2} @ A u N P$ substrate
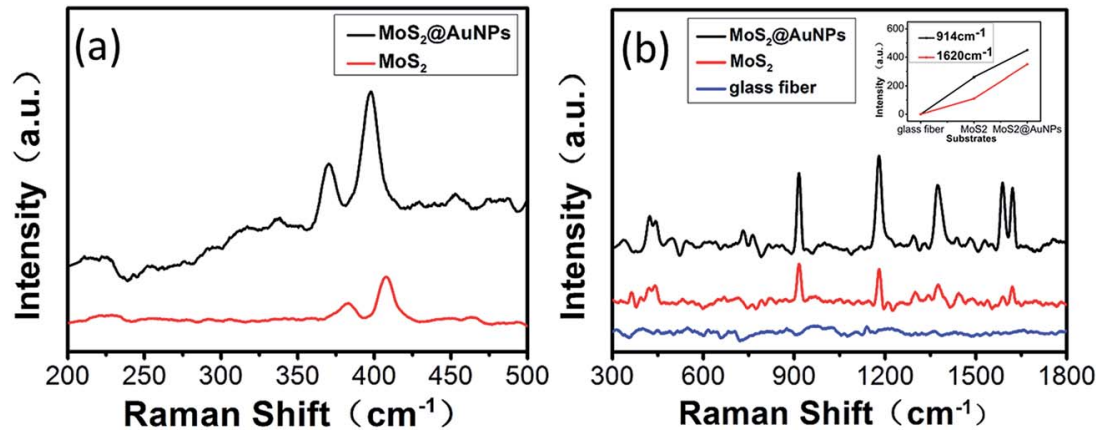

Fig. 3 (a) Raman spectrum of MoS 2 on GF membranes (red line) and GF-MoS 2 (AuNPs (black line). The peaks at $377 \mathrm{~cm}^{-1}$ and $408 \mathrm{~cm}^{-1}$ are attributed to $E_{2 g}^{1}$ and $A_{1 g}$, respectively. Raman signals of the $M_{o S}$ molecules slightly increase when it is decorated with AuNPs. (b) The Raman spectrum of $\mathrm{CV}$ with a concentration of $10^{-4} \mathrm{M}$ on different SERS-active substrates. Both GF-MoS 2 (aANPs substrate and substrate only with $\mathrm{MoS}_{2}$ have SERS effects for CV, and the former shows better performance. The inset is the Raman intensity of the CV molecules at $914 \mathrm{~cm}^{-1}$ and $1620 \mathrm{~cm}^{-1}$ for various SERS-active substrates. 

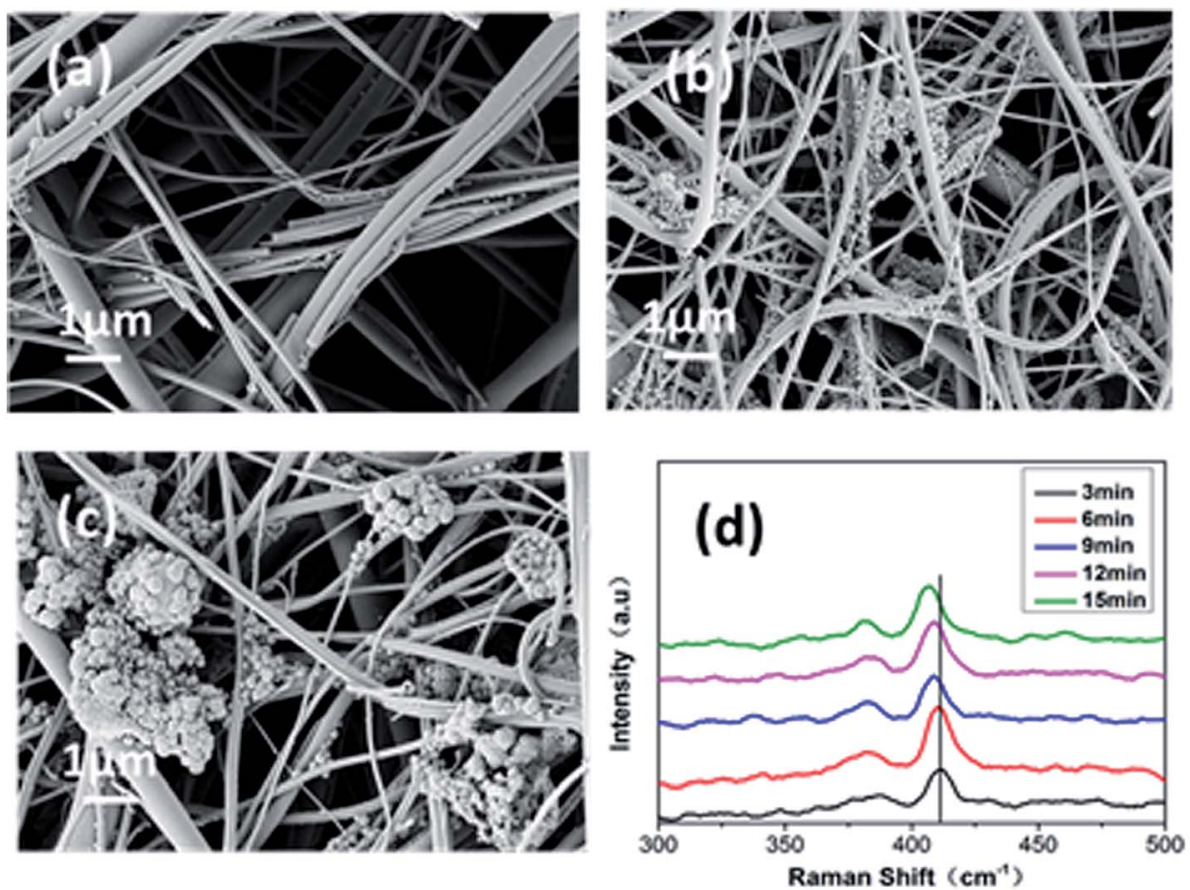

Fig. 4 SEM images of the GF-MoS 2 (aAuNP substrate obtained at different reaction times: (a) 3 min; (b) 9 min, and (c) 15 min. (d) The Raman spectrum for $\mathrm{MOS}_{2}$ treatment at $3 \mathrm{~min}, 6 \mathrm{~min}, 9 \mathrm{~min}, 12 \mathrm{~min}$, and $15 \mathrm{~min}$.

obtained at various reaction times. As shown in Fig. 4a, when the reaction time is $3 \mathrm{~min}$, only few and small nanoparticles are formed as seeds on the surface of the GF membranes or at the gap between fibers. As the time increases, AuNPs become larger in size and more in number, and a high-density AuNP-decorated $\mathrm{MoS}_{2}$ nanocomposite is obtained at $9 \mathrm{~min}$; however, most of the AuNPs are distributed in the intersection of fibers (Fig. 4b). When the reaction time is increased to $15 \mathrm{~min}$, AuNPs grow larger and become aggregated (Fig. 4c) because with continuous reactions between $\mathrm{HAuCl}_{4}$ and $\mathrm{MoS}_{2}$, Au ions in the solution become reduced by the active nucleation sites and result in anchoring of AuNPs on these sites. Several AuNPs come together to form a large particle; this leads to the exposure of fibers. With the continuous reaction between $\mathrm{MoS}_{2}$ and $\mathrm{HAuCl}_{4}$, the number of layers of the $\mathrm{MoS}_{2}$ films changed. Fig. 4d shows the Raman spectrum of $\mathrm{MoS}_{2}$ with different reaction times. Note that the $\mathrm{A}_{1 \mathrm{~g}}$ peak undergoes a blue shift, whereas the $\mathrm{E}_{2 \mathrm{~g}}^{1}$ peak undergoes a slightly red shift; this suggests that the number of $\mathrm{MoS}_{2}$ layers decreases with the increase in the reaction time. This may be because S-Mo-S bonding in $\mathrm{MoS}_{2}$ is not strong enough and the interlayer interaction between $\mathrm{MoS}_{2}$ layers is weak. Continuous chemical reactions between $\mathrm{MoS}_{2}$ and $\mathrm{HAuCl}_{4}$ lead to defects in $\mathrm{MoS}_{2}$ and then decrease its thickness.

To explore the SERS performance of the GF-MoS ${ }_{2} @ A u N P$ substrates at different reaction times, the Raman spectrum of all substrates towards $\mathrm{CV}$ with a concentration of $10^{-4} \mathrm{M}$ is shown in Fig. 5a. The time of the reaction between $\mathrm{MoS}_{2}$ and $\mathrm{HAuCl}_{4}$ has a significant impact on the SERS properties of the GF-MoS $\mathrm{M}_{2}$ @AuNP substrate. The SERS intensity is weaker when the reaction time is short. The reason is that the number of AuNPs is small, and hence, the hot spots on the substrate are very less. The SERS intensity becomes much stronger with the increase in the reaction time because AuNPs are dense and less aggregated, which can generate more hot spots. The SERS effect of the substrate depends on the AuNP size and density. With further increase in the reaction time, AuNPs seriously aggregate and move far away from other gold nanoclusters; this reduces the number of hot spots and weaken the SERS intensity. ${ }^{29}$ According to previous studies, the SERS performance is also affected by the number of $\mathrm{MoS}_{2}$ layers; thinner $\mathrm{MoS}_{2}$ shows better SERS performance. As the $\mathrm{CV}$ peak near $914 \mathrm{~cm}^{-1}$ is more representative and its intensity is very sensitive, according to the spectra, it is selected as the representative peak to evaluate the sensitivity of different substrates. Fig. 5b demonstrates that the GF-MoS $\mathrm{M}_{2} @ \mathrm{AuNP}$ substrate produces the best effect of Raman enhancements when the reaction time is 9 min not only because it has dense particles but also because it has a thinner $\mathrm{MoS}_{2}$ layer.

Although the extension of the reaction time can enhance the SERS effect of the substrate to a certain extent, continuous reactions between $\mathrm{HAuCl}_{4}$ and $\mathrm{MoS}_{2}$ reduce the number of hot spots and affect the Raman enhancement of the substrate. Through further research, we found that multiple repetitions of chemical reactions between $\mathrm{MoS}_{2}$ and $\mathrm{HAuCl}_{4}$ could control the sizes of the deposited nanoparticles and greatly enhance the SERS effect. We proposed a power-free method to densely and uniformly deposit AuNPs on glass fiber; this method is similar to the successive ionic layer absorption and reaction (SILAR) method. ${ }^{30}$ This method provided control over the size of particles via a number of chemical treatment cycles. Compared with other methods, in this method, multiple repetitions of chemical reactions produce evenly distributed AuNPs and reduce the polymerization of AuNPs. We again chose $3 \mathrm{~min}$ as a time interval 

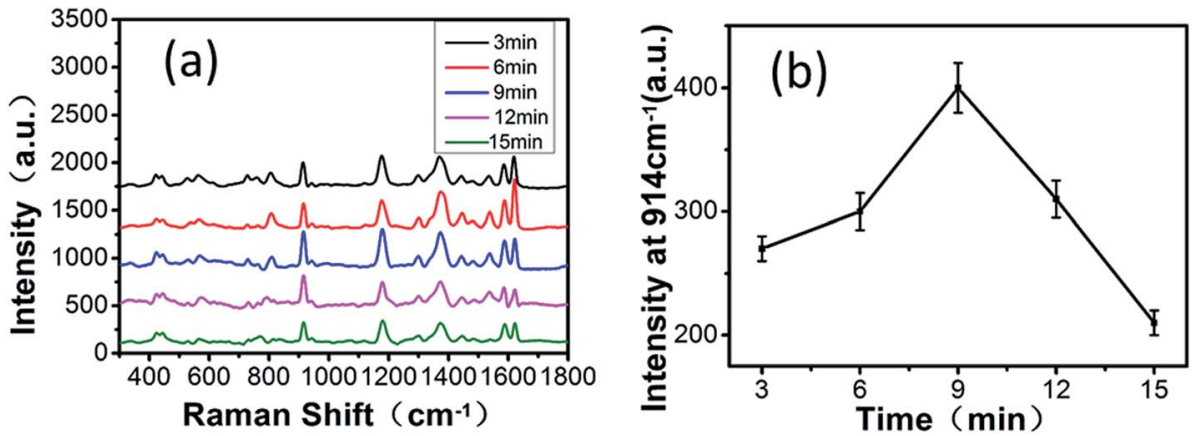

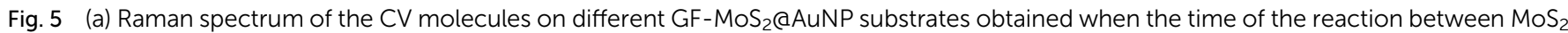
and $\mathrm{HAuCl}_{4}$ is increased from 3 to $15 \mathrm{~min}$. As time increases, the SERS effect of the substrate changes constantly. (b) Raman intensity of CV molecules at $914 \mathrm{~cm}^{-1}$ for various substrates. The GF-MoS 2 AANP substrate shows the best SERS performance when the reaction time is 9 min.

again to achieve multiple AuNP deposition. For the first cycle, the $\mathrm{MoS}_{2}$ substrate was immersed in $\mathrm{HAuCl}_{4}$ solution and treated for $3 \mathrm{~min}$; then, it was removed and rinsed with deionized water to wash out the non-reacted residues to obtain the GF-MoS $\mathrm{S}_{2} @ A u N P-$ 1 substrate. Then the GF-MoS ${ }_{2} @ A u N P-1$ nanocomposite was immersed in $\mathrm{HAuCl}_{4}$ solution again for the same reaction time to

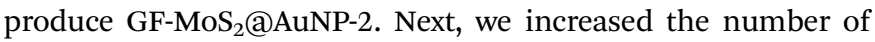
chemical treatment cycles to obtain GF-MoS 2 @AuNP-3, GF$\mathrm{MoS}_{2} @ A u N P-4$, and GF-MoS $@$ @AuNP-5. Fig. 6a is the SEM image of the GF-MoS $\mathrm{S}_{2} @ A u N P-2$. AuNPs on GF-MoS $\mathrm{S}_{2} @ A u N P-3$ are significantly increased, but nanoparticles are gathered in the intersection of fibers and inhomogeneously distributed (Fig. 6b). Compared to Fig. 4b, which is an SEM image obtained at $9 \mathrm{~min}$, Fig. 6c shows GF-MoS $\mathrm{M}_{2} @$ AuNP-4 with high density and uniform distribution of AuNPs. AuNPs begin to cluster (red box in Fig. 6d) and the surface of the GF membranes is exposed as described earlier. The AuNPs produced in the first cycles act as seeds and their quantity and diameters are gradually increased via a synthesis reaction that occurred at the seeds through multiple repetitions. The electrostatic repulsion of the charged surface can make the AuNPs stable in solution. However, the lack of sufficient surface charge and stabilizing agent for our products do not prevent the AuNPs from aggregating. Compared with the SILAR method, via the proposed method, we synthesized a layered structure of $\mathrm{MoS}_{2}$ sheets with AuNPs on GF membranes. GF membranes with a more uniform structure and higher temperature resistance compared to paper can be widely applied in rapid detection and analysis under on-site conditions. We
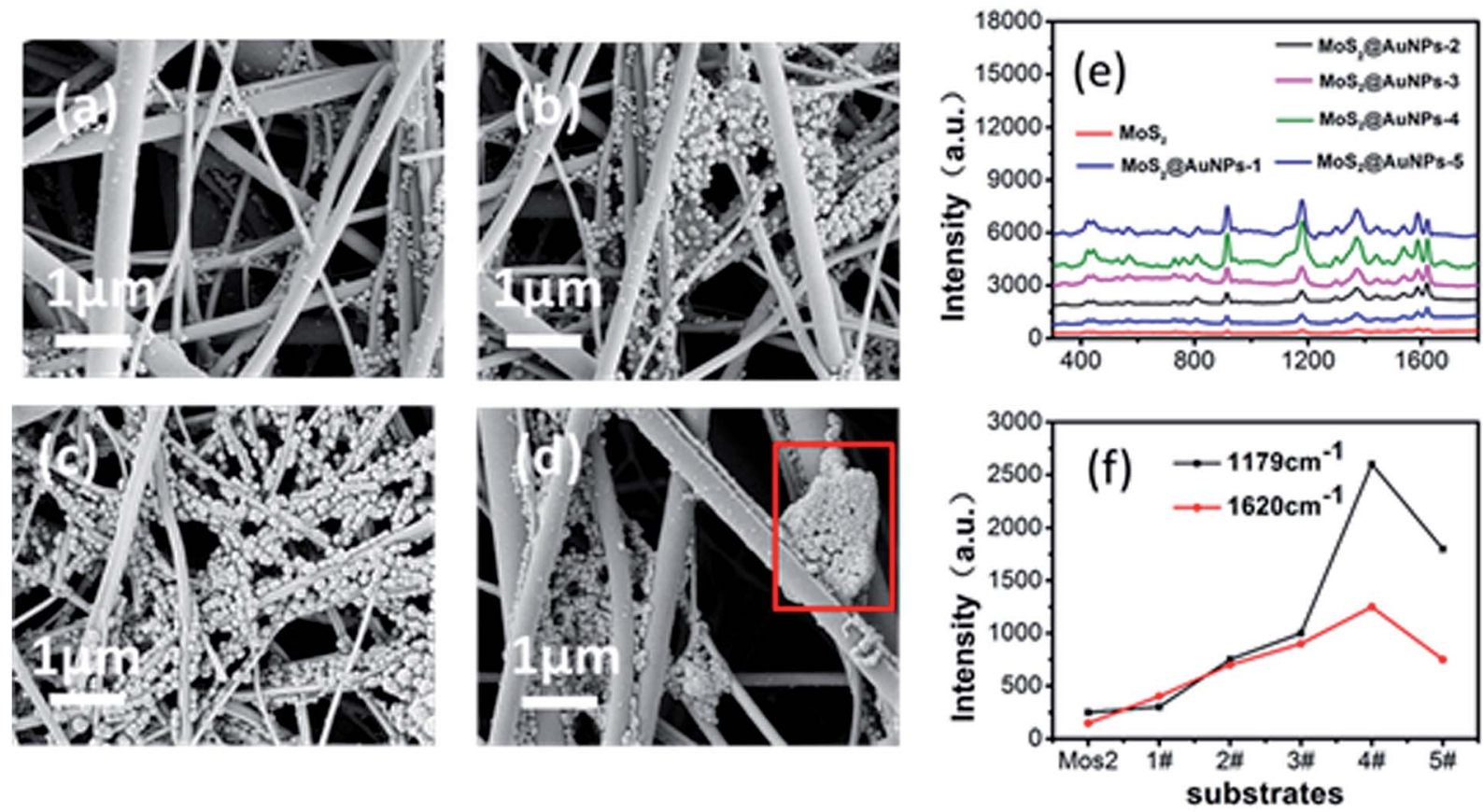

Fig. 6 (a)-(d) SEM image of MoS 2 (AANP-2, MoS 2 @AuNP-3, MoS $2 @ A A u N P-4$, and MoS 2 AAuNP-5. (e) Raman spectrum of CV with a concentration of $10^{-4} \mathrm{M}$ on different substrates. (f) Raman intensity of the $\mathrm{CV}$ molecules at $1179 \mathrm{~cm}^{-1}$ and $1620 \mathrm{~cm}^{-1}$ for various substrates. Both Raman peaks show highest intensity for the GF-MoS 2 aAuNP-4 substrate. 
produced $\mathrm{MoS}_{2}$ layers on GF membranes primarily via the CVD method rather than directly synthesizing metal nanoparticles on the substrate. In addition to the Raman enhancement effect, $\mathrm{MoS}_{2}$ can firmly adsorb metal nanoparticles and reduce background fluorescence during detection. $\mathrm{MoS}_{2}$ shows great ability in redox chemistry, and it can directly react with $\mathrm{HAuCl}_{4}$ to allow a straightforward and green synthesis of AuNPs. Unbound sulfurs act as sites in Au nuclei seeding and growth, and the total rate of assembly of AuNPs is governed by the rate of diffusion of $\mathrm{Au}$ ions into the $\mathrm{MoS}_{2}$ surface and the rate of Au incorporation into the crystalline particles. When $\mathrm{MoS}_{2}$ film is repeatedly immersed in solution, the introduction of high defect density facilitates a large diffusion coefficient. This reduces the polymerization of nanoparticles and provides more hot spots. ${ }^{22}$ For comparison, the CV molecules $\left(10^{-4} \mathrm{M}\right)$ were also deposited onto the 9 min-treated substrate and five substrates above-mentioned for Raman detection under the same condition (Fig. 6e). Compared with the Raman spectroscopy of the 9 min-treated substrate, the enhancement effect of substrates is greatly improved by the number of chemical treatment cycles; this indicates that multiple AuNP deposition can greatly increase the SERS effect of the substrates. To compare the Raman enhancement magnitude, we chose the SERS intensity of $\mathrm{CV}$ on different substrates at the selected low obvious Raman peaks $\left(1179 \mathrm{~cm}^{-1}\right.$ and $1620 \mathrm{~cm}^{-1}$ ). The data are obtained from at least five different locations of the substrate. From Fig. 6f, we discovered that on increasing the number of chemical treatments cycles, the SERS activity was enhanced until four cycles. Moreover, both Raman peaks show highest intensity on the GF-MoS ${ }_{2} @ A u N P-4$ substrate, indicating that this substrate shows the best SERS performance for $\mathrm{CV}$.

GF- $\mathrm{MoS}_{2} @ A u N P-4$ substrates are exposed to CV and TB solutions of different concentrations, and Raman spectra of CV and TB are shown in Fig. 7a and b, respectively. The blank spectra are the Raman spectra where there is no $\mathrm{CV}$ or $\mathrm{TB}$, and there are only two Raman peaks belonging to $\mathrm{MoS}_{2}$. It can be observed that the detection limit of GF-MoS ${ }_{2} @ A u N P$ substrate for $\mathrm{CV}$ is $10^{-8} \mathrm{M}$. By contrast, the limit for TB detection is lower than $10^{-7} \mathrm{M}$ as TB has good signal-to-noise ratio and its main peaks are still observable at the Raman spectra of $10^{-7} \mathrm{M}$. Hence, it is obvious that the GF-MoS $\mathrm{S}_{2}$ @AuNP-4 substrate is able to improve the detection sensitivity for both CV and TB molecules. Considering the fact that the surface enhancement factor (EF) is one of the most important parameters for characterizing the SERS effect, in this study, the EF of the GNP film is calculated based on the following equation:

$$
\mathrm{EF}=\left(I_{\mathrm{SERS}} / I_{\mathrm{Raman}}\right)\left(C_{\mathrm{Raman}} / C_{\mathrm{SERS}}\right)
$$

where $I_{\text {SERS }}$ is the Raman intensity obtained for the SERS substrate at a certain concentration; $I_{\text {Raman }}$ is the Raman intensity obtained under non-SERS conditions; $C_{\text {Raman }}$ and $C_{\text {SERS }}$ represent the CV solution concentrations deposited onto the GF and the GF-MoS $@$ @AuNP-4 substrate, respectively. Herein, we chose the $1620 \mathrm{~cm}^{-1}$ peak of the $\mathrm{CV}$ molecule as the reference peak. Using the data shown in Fig. 7a, an EF of $1.3 \times$ $10^{6}$ is determined $\left(C_{\mathrm{SERS}}=10^{-8} \mathrm{M}\right.$ and $\left.C_{\text {Raman }}=10^{-2} \mathrm{M}\right)$. To further investigate the SERS performance of the GF-
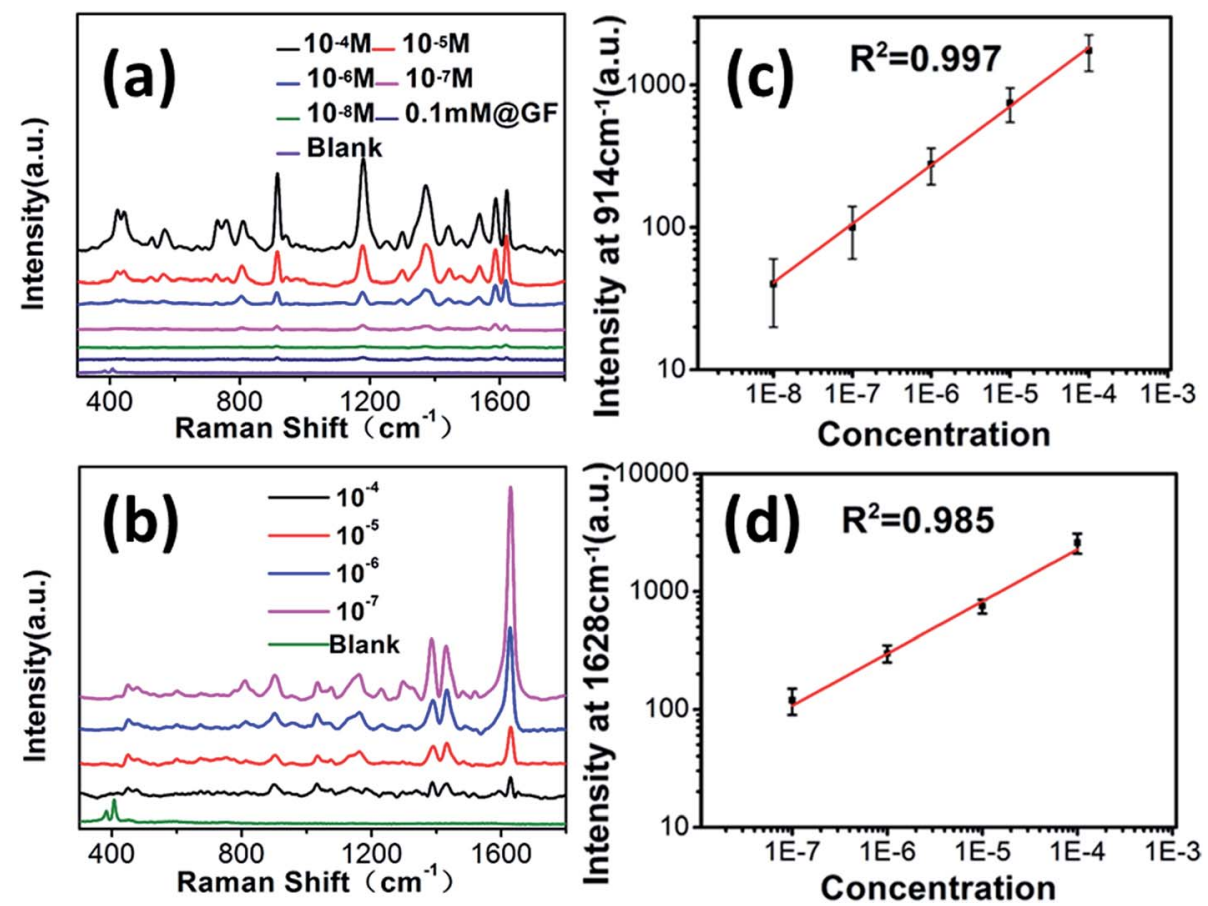

Fig. 7 (a) SERS spectra of the CV molecules with different concentrations. (b) SERS spectra of the TB molecules with different concentrations. (c) Raman intensity of the CV molecules at $914 \mathrm{~cm}^{-1}$ for various concentrations. (d) Raman intensity of the TB molecules at $1631 \mathrm{~cm}^{-1}$ for various concentrations. 
$\mathrm{MoS}_{2} @ A u N P-4$ substrate, Raman peaks located at $914 \mathrm{~cm}^{-1}$ and $1631 \mathrm{~cm}^{-1}$ are chosen as signatures to determine the concentration of CV and TB in the samples. From Fig. 7c, it can be seen that the Raman intensity of CV molecules at $914 \mathrm{~cm}^{-1}$ is linear when the SERS signal ranges from $10^{-4}$ to $10^{-8} \mathrm{M}$. In this case, the value of $R^{2}$ is 0.997 . Similarly, Fig. 7d shows that the Raman intensity of TB molecules at $1631 \mathrm{~cm}^{-1}$ is linear when the SERS signal ranges from $10^{-4}$ to $10^{-7} \mathrm{M}$ and $R^{2}$ reaches 0.985 . Furthermore, it can be seen that for both CV and TB molecules, the intensity of peaks decreases linearly as the concentration

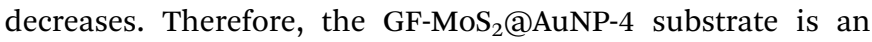
effective approach that can be applied to quantitatively detect both the CV and TB molecules.

For application purposes, SERS substrates are required to have good stability and reproducibility during the Raman measurements. Fig. 8a-c show the SERS spectra of $10^{-5} \mathrm{M} \mathrm{CV}$ adsorbed on the GF-MoS ${ }_{2} @ A u N P-4$ substrate, which are obtained for five different spots on three different parts of the structure. As seen, although there is a slight variability of the intensity, the characteristic scattering peaks of $\mathrm{CV}$ can be detected on all spots. To investigate the stability of the GF$\mathrm{MoS}_{2} @$ AuNP-4 substrate, the CV concentration of $10^{-5} \mathrm{M}$ is selected to compare the stability of the substrates. The Raman intensity is measured every five days at room temperature. As shown in Fig. 8d, the Raman intensity of the peaks at $914 \mathrm{~cm}^{-1}$ and $1620 \mathrm{~cm}^{-1}$ is attenuated with the increase in the number of days, and the attenuation of the Raman intensity becomes weak after 10 days. The average intensity of the peak at $914 \mathrm{~cm}^{-1}$ is dropped by $36 \%$ and the average intensity at $1620 \mathrm{~cm}^{-1}$ is decreased only by $28 \%$. Through this analysis, we can conclude that this substrate shows high repeatability and long-term stability. Thus, the GF-MoS ${ }_{2}$ @AuNP-4 substrate is a good candidate for practical analytical applications of SERS.

The previous substrate synthesized via the SILAR method was used only as a SERS platform; however, the GF-MoS ${ }_{2}$ @AuNP-4 substrate shows great potential in excellent paper chromatographic separation and is a sensitive SERS substrate for biological and chemical detection. Rapid screening of mixed small molecules has been achieved by the method of paper chromatographic separation. GF membranes are porous, and ethanol as a mobile phase can quickly spread under the action of capillary tension. As CV and TB molecules have different distribution coefficients in alcohol and water, with the spread of alcohol, they move at different speeds on the fiber to achieve separation. Colorimetric detection of dye molecules (CV, TB, and their mixed solution $10^{-4} \mathrm{M}$ ) after paper separation on GF membranes is shown in Fig. 9a. Points 1 and 2 are the CV and TB molecules after they moved up with the spread of alcohol. Mixed molecules after separation appear at points 3 and 4, respectively. On comparing the separated molecules with $\mathrm{CV}$ and TB, it was observed that points 1 and 3 have similar color and displacement, and the same situation also occurs for points 2 and 4; this indicates that the mixed molecules of TB and CV can be separated on GF membranes via paper separation. However, the separated molecules cannot be detected by the Raman microscopy system because of their low concentrations.

The Raman spectra obtained when the mixed solution of CV and TB is spotted on the $\mathrm{MoS}_{2}$ substrate using a capillary tube are shown in Fig. 9b. Complex spectra appeared due to the presence of two molecules; therefore, the type of the molecule
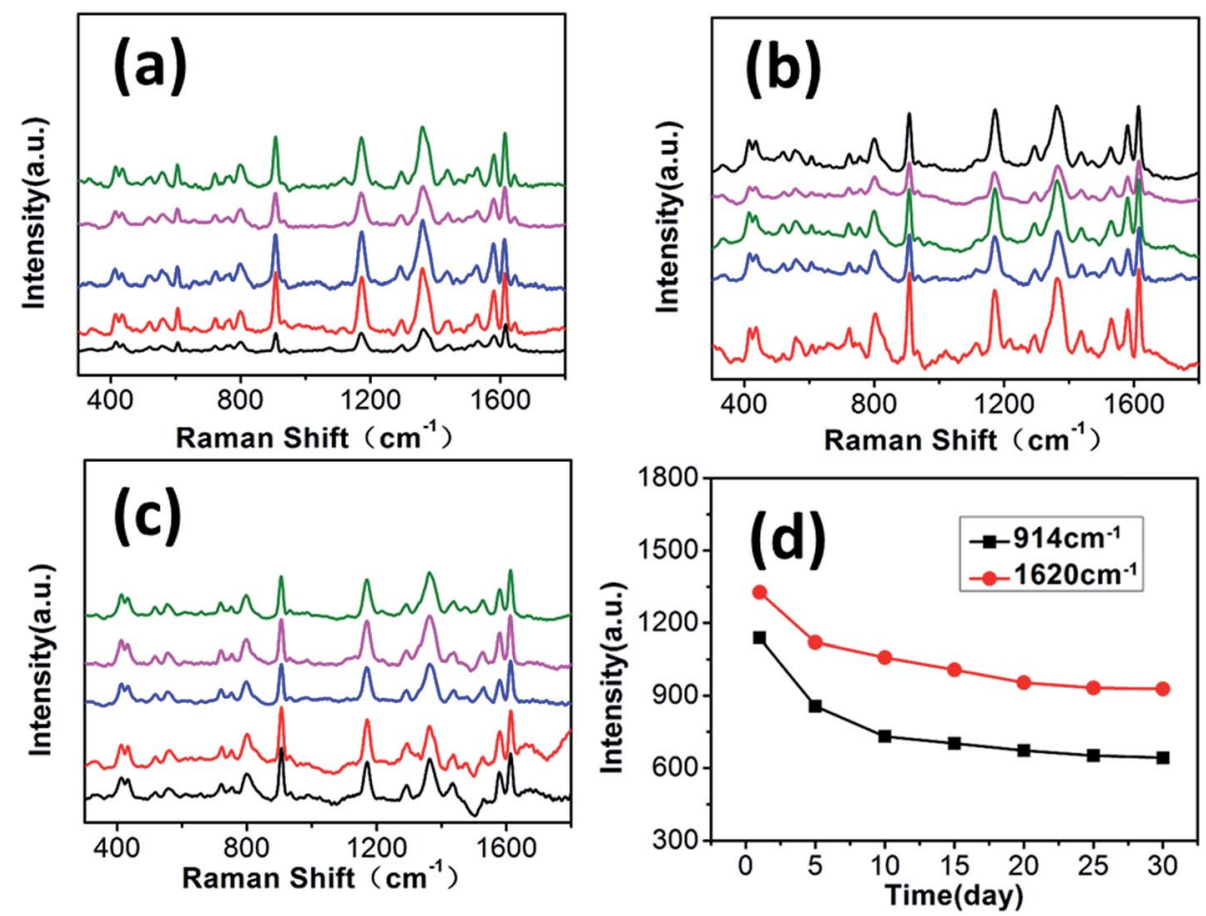

Fig. 8 (a)-(c) SERS spectra of CV $\left(10^{-5} \mathrm{M}\right)$ on the GF-MoS 2 (AANP-4 substrate recorded for five different spots on three different parts. (d) The Raman intensity changes of the peaks at $914 \mathrm{~cm}^{-1}$ and $1620 \mathrm{~cm}^{-1}$ with time. 

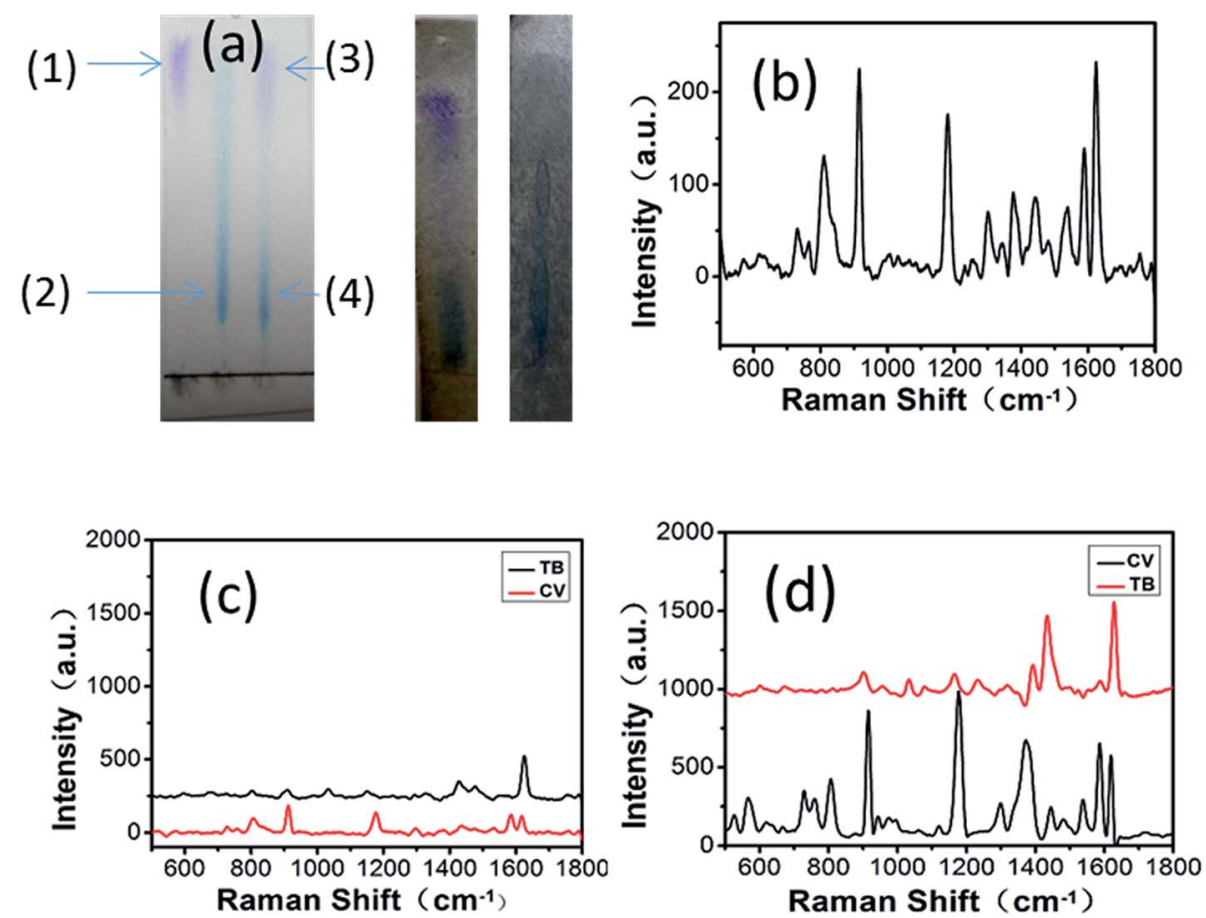

Fig. 9 (a) Colorimetric detection of dye molecules (CV, TB, and their mixed solution) after paper separation and images of the mixed solution after separation on the $\mathrm{MoS}_{2}$ and GF-MoS 2 (AuNP-4 substrates. The molecules of CV and TB are at the (1) and (2) positions. And the separated molecules of CV and TB from mixed solution are at the (3) and (4) positions. (b) Raman spectra of the mixed solution. (c) and (d) Raman spectra of the CV and TB molecules after paper separation on the MoS 2 and GF-MoS 2 AuNP-4 substrates.

cannot be determined. When mixed molecules are clearly distinguished after paper separation on the $\mathrm{MoS}_{2}$ substrate, the two types of molecules can be determined separately and their Raman spectra can be obtained (Fig. 9c). According to the location of the characteristic peaks of $\mathrm{CV}$ and TB, it is clear that the two Raman spectra belong to CV and TB, respectively. The third image in Fig. 9a shows the images of the mixed solution after paper separation on the GF-MoS ${ }_{2} @ A u N P-4$ substrate. Compared with the second image, the distance between the two points after separation on the GF-MoS $@$ @AuNP-4 substrate is less. The reasons may be that separated molecules reside near the nanogap-rich AuNPs, which allow the plasmonic enhancement of the SERS signals. We can observe that Raman signals in Fig. 9d are much stronger than those in Fig. 9c because of the presence of AuNPs. Mixed small molecules can be successfully distinguished over both substrates and also detected by SERS measurement after paper separation. However, the GF$\mathrm{MoS}_{2} @ A u N P-4$ substrate can achieve more sensitive detection of small molecules; thus, it can be used to separate and detect molecules with lower concentrations. When a lower concentration $\left(10^{-8} \mathrm{M}\right)$ of the mixed solution is separated on the substrate, although the color cannot be resolved, we can still detect their Raman spectra at their corresponding positions.

\section{Conclusion}

In conclusion, we obtained an efficient SERS-active substrate by synthesizing a GF-MoS $\mathrm{S}_{2} @ A u N P$ substrate with uniform and high density of AuNPs. Layered $\mathrm{MoS}_{2}$ was applied to synthesize the AuNPs that enhanced the SERS signal via chemical adsorption. AuNPs are used to obtain the localized electric field enhancement. The GF membranes, which cannot be easily destroyed due to their thermal stability, are used to separate the mixed molecules. These GF-MoS $\mathrm{MOAuNP}_{2}$ hybrid substrates show a huge potential for an excellent paper-based separation and are SERS-active substrates.

\section{References}

1 C. Yang, C. Zhang, Y. Huo, S. Jiang, H. Qiu, Y. Xu, X. Li and B. Man, Carbon, 2016, 98, 526-533.

2 W. L. Barnes, A. Dereux and T. W. Ebbesen, Nature, 2003, 424, 824-830.

3 K. Kneipp, H. Kneipp and J. Kneipp, Acc. Chem. Res., 2006, 39, 443-450.

4 M. M. Maye, J. Luo, L. Han and C.-J. Zhong, Nano Lett., 2001, 1, 575-579.

5 W. W. Yu and I. M. White, Analyst, 2013, 138, 3679-3686.

6 C. Mueller, W. Weinmann, S. Dresen, A. Schreiber and M. Gergov, Rapid Commun. Mass Spectrom., 2005, 19, 13321338.

7 K. S. Minioti, C. F. Sakellariou and N. S. Thomaidis, Anal. Chim. Acta, 2007, 583, 103-110.

8 N. Gogoi, M. Barooah, G. Majumdar and D. Chowdhury, ACS Appl. Mater. Interfaces, 2015, 7, 3058-3067.

9 M. A. Farajzadeh and M. R. A. Mogaddam, Anal. Chim. Acta, 2012, 728, 31-38. 
10 B. Buszewski and S. Noga, Anal. Bioanal. Chem., 2012, 402, 231-247.

11 F. Karabiber, J. L. McGinnis, O. V. Favorov and K. M. Weeks, $R N A$, 2013, 19, 63-73.

12 H. Wang, J. Liu, R. G. Cooks and Z. Ouyang, Angew. Chem., 2010, 122, 889-892.

13 H. Jung, M. Park, M. Kang and K.-H. Jeong, Light: Sci. Appl., 2016, 5, e16009.

14 S. Jiang, J. Guo, C. Zhang, C. Li, M. Wang, Z. Li, S. Gao, P. Chen, H. Si and S. Xu, RSC Adv., 2017, 7, 5764-5773.

15 H. Qiu, Z. Li, S. Gao, P. Chen, C. Zhang, S. Jiang, S. Xu, C. Yang and H. Li, RSC Adv., 2015, 5, 83899-83905.

16 J. Zhao, Z. Zhang, S. Yang, H. Zheng and Y. Li, J. Alloys Compd., 2013, 559, 87-91.

17 S. Su, C. Zhang, L. Yuwen, J. Chao, X. Zuo, X. Liu, C. Song, C. Fan and L. Wang, ACS Appl. Mater. Interfaces, 2014, 6, 18735-18741.

18 E. Boisselier and D. Astruc, Chem. Soc. Rev., 2009, 38, 17591782.

19 S.-W. Joo, Vib. Spectrosc., 2004, 34, 269-272.

20 Y. W. Chen, T. Y. Liu, P. J. Chen, P. H. Chang and S. Y. Chen, Small, 2016, 12, 1458-1468.
21 S. Najmaei, A. Mlayah, A. Arbouet, C. Girard, J. Léotin and J. Lou, ACS Nano, 2014, 8, 12682.

22 A. M. Schwartzberg, C. D. Grant, A. Wolcott, C. E. Talley, T. R. Huser, R. Bogomolni and J. Z. Zhang, J. Phys. Chem. $B, 2004,108,19191-19197$.

23 C. M. Cobley, J. Chen, E. C. Cho, L. V. Wang and Y. Xia, Chem. Soc. Rev., 2011, 40, 44-56.

24 C. Yang, Y. Xu, C. Zhang, Z. Sun, C. Chen, X. Li, S. Jiang and B. Man, Nanoscale Res. Lett., 2014, 9, 394.

25 X. Fang, S. Wei and J. Kong, Lab Chip, 2014, 14, 911-915.

26 H. X. Gu, D. W. Li, L. Xue, Y. F. Zhang and Y. T. Long, Analyst, 2015, 140, 7934.

27 K. Zhang, J. Zhao, H. Xu, Y. Li, J. Ji and B. Liu, ACS Appl. Mater. Interfaces, 2015, 7, 16767.

28 T. Sreeprasad, P. Nguyen, N. Kim and V. Berry, Nano Lett., 2013, 13, 4434-4441.

29 S. Shao, Z. Chi, L. Yuwen, C. Jie, X. Zuo, X. Liu, C. Song, C. Fan and L. Wang, ACS Appl. Mater. Interfaces, 2014, 6, 18735.

30 W. Kim, Y. H. Kim, H. Park and S. Choi, ACS Appl. Mater. Interfaces, 2015, 7, 27910. 